\title{
DAS28 score vs. ultrasound examination for assessment of rheumatoid arthritis disease activity: comparison and discussion of pros and cons
}

\author{
Tacjana Anna Barczyńska ${ }^{1}$, Marta Dura², Einat Blumfield ${ }^{3}$, Małgorzata Węgierska ${ }^{1}$, \\ Pawel Żuchowski ${ }^{4}$, Arnika Wilińska-Jankowska ${ }^{1}$, Sławomir Jeka ${ }^{1}$
}

${ }^{1}$ Clinic of Rheumatology and Connective Tissue Diseases, University Hospital Nr 2 Dr. Jan Biziel, Bydgoszcz, Poland ${ }^{2}$ NZOZ Nasz Lekarz, Torun, Poland

${ }^{3}$ Jacobi Medical Center, Albert Einstein College of Medicine, Bronx, NY, USA

${ }^{4}$ Independent Section of Scientific Research Coordination and Didactic Activity, University Hospital Nr 2 Dr. Jan Biziel, Bydgoszcz, Poland

\begin{abstract}
Rheumatoid arthritis (RA) is a chronic systemic connective tissue disease which is characterized by symetrical multiple joints involvement and extra-articular symptoms. Current EULAR diagnostic criteria for RA include disease activity parameters, such as erythrocyte sedimentation rate (ESR) and C-reactive protein (CRP), which are used to calculate disease activity scores, including DAS and DAS28. Recently attempts have been made to assess disease activity using imaging diagnostic modalities, such as magnetic resonance imaging (MRI) and ultrasonography (US). Due to significant progress in therapy effectiveness and early RA diagnosis possibility, imaging modalities become in creasingly meaningful and many clinical trials confirm their usefulness. However, there are no consistent criteria for objective assessment of therapy effectiveness based on US. Moreover, it is not US availability that limits its common use, but rather significant variability between operators. This is why US remains only an additional tool to assess therapy efficacy with regard to DAS/DAS28 index.
\end{abstract}

Key words: ultrasonography, rheumatoid arthritis, DAS28.

\section{Introduction}

Developing a reliable method for assessment of rheumatoid arthritis (RA) progression, and effectiveness of administered therapy, is a time-consuming process. Proposed methods often yield disputable results [1]. Steinbrocker's criteria, which are based on radiographs, became widely accepted early on. These criteria were developed in 1949 and were included in the New York diagnostic criteria for RA in 1981. However, they are not reliable for assessment of the disease in its early stage when joint lesions are not evident on radiographs. More precise methods for disease progression monitoring, and assessment of therapeutic effectiveness, were developed in the 1970s by Sharp, Larsen and Dale [2,3]. The currently applicable ACR/ EULAR 2010 criteria are more useful in detection of early RA stages, but do not rely on imaging studies [4]. The Health Assessment Questionnaire (HAQ) is another method to assess therapy effectiveness, and is being used increasingly.

Nevertheless, the most widely used method for RA activity evaluation is the Disease Activity Score (DAS) and its simplified version - DAS28. 


\section{DAS scores}

The DAS score was developed in Nijmegen, the Netherlands. The development began in 1983 for the purpose of assessment of RA activity for a clinical study and was continued for several years thereafter. In 1985 the first patients with early RA, for whom the score was designed, were included in the study.

DAS/DAS28 calculation formulas include the number of tender and swollen joints, self-assessment of health using the visual-analog scale (VAS), and erythrocyte sedimentation rate (ESR) or C-reactive protein (CRP).

Naturally, the above parameters do not contribute equally to the final DAS/DAS28 result. The numeric contributions of the different parameters are expressed by various mathematical functions - from the simplest linear function (VAS) to a logarithmic function (ESR or (RP). As a result the contribution of each parameter to the final DAS/DAS28 score is different. Similarly, changes of parameter values during therapy monitoring affect the score differently.

Due to its complex mathematical formulation, DAS/ DAS28 is generally calculated using a dedicated calculator, and clinicians obtain the final score often without understanding the significance of the individual contribution of each parameter. In the authors' opinion it would be beneficial to understand the significance of each parameter in forming the final DAS/DAS28 score prior to comparing it to other currently available diagnostic modalities. To simplify the analysis, we will further use only the DAS28 score, which is currently used in clinical practice more commonly than the DAS score because it requires assessment of fewer joints (only 28 joints are assessed for the DAS28 score, while 53 joints

Table I. Disease activity measured using DAS28

\begin{tabular}{|lc|}
\hline Disease activity & DAS28 value \\
\hline Remission & DAS28 $\leq 2.6$ \\
\hline Low disease activity & $2.6<$ DAS28 $\leq 3.2$ \\
\hline Moderate disease activity & $3.2<$ DAS $28 \leq 5.1$ \\
\hline High disease activity & $5.1<$ DAS 28 \\
\hline
\end{tabular}

are assessed for tenderness and 44 joints are assessed for swelling in the DAS score). Additionally, we will discuss only the DAS28 score including ESR; calculations for DAS28 using CRP can be done similarly (like ESR, CRP'S contribution to the score is expressed by a logarithmic function).

\section{DAS28 - significance of individual parameters}

The formula for the DAS28 score is as follows [2]:

$0.56 \sqrt{(T J C 28)}+0.28 \sqrt{(S J C 28)}+0.7 \ln (E S R)+0.014 G E$

The following symbols were used in the formula:

TJC28 - tender joint count from 28 examined,

SJC28 - swollen joint count from 28 examined,

ESR - erythrocyte sedimentation rate $[\mathrm{mm} / \mathrm{h}]$,

GE - patient's self-assessed general health expressed on a $100 \mathrm{~mm}$ VAS scale.

Based on the DAS28 score, patients can be categorized into four groups (Table I). There are minimal differences in the score between the three active groups. This enables evaluation of the efficacy of administered therapy (Table II). Figure 1 presents graphs of the functions for all contributing parameters.

The parameter of general health assessment using the VAS scale is subjective, so its contribution to the DAS28 score is relatively small, as it is expressed by a linear function in formula [1]. Nevertheless, in extreme cases (where the change in the VAS scale is $\geq 40 \mathrm{~mm}$, translating to a change of $\geq 0.56$ in the DAS28 score) this may lead to distortion in the assessment of disease activity (e.g. rendering administered therapy effective when it is not). Therefore there is a modified version of the DAS28 score which includes only the tender and swollen joint count and ESR and excludes the general health parameter [3].

The effect of ESR on the DAS28 score is expressed as a logarithmic function. This means that there is a rapid increase in the score with small increments in the lower range of ESR values (i.e. 0-40) and a slower increase in the higher range of ESR values.

Consequently, ESR changes above the value of 40 have only a minor effect on DAS28 score change.

Table II. EULAR therapy response criteria using DAS28

\begin{tabular}{|c|c|c|c|}
\hline \multirow[t]{2}{*}{ Present DAS28 } & \multicolumn{3}{|c|}{ DAS28 improvement } \\
\hline & $>1.2$ & $>0.6$ and $\leq 1.2$ & $\leq 0.6$ \\
\hline$\leq 3.2$ & good response & moderate response & no response \\
\hline$>3.2$ and $\leq 5.1$ & moderate response & moderate response & no response \\
\hline$>5.1$ & moderate response & no response & no response \\
\hline
\end{tabular}


A

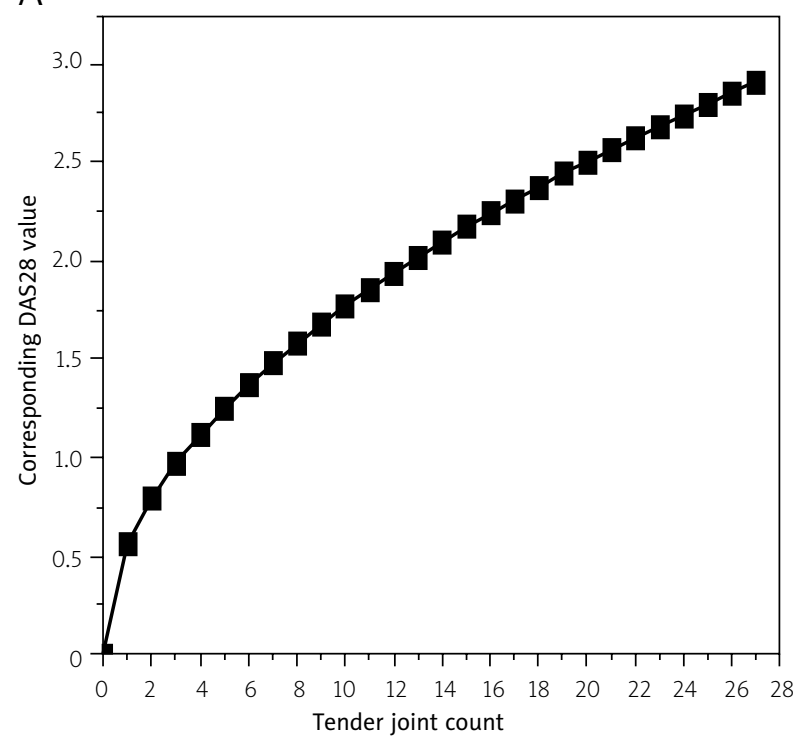

C

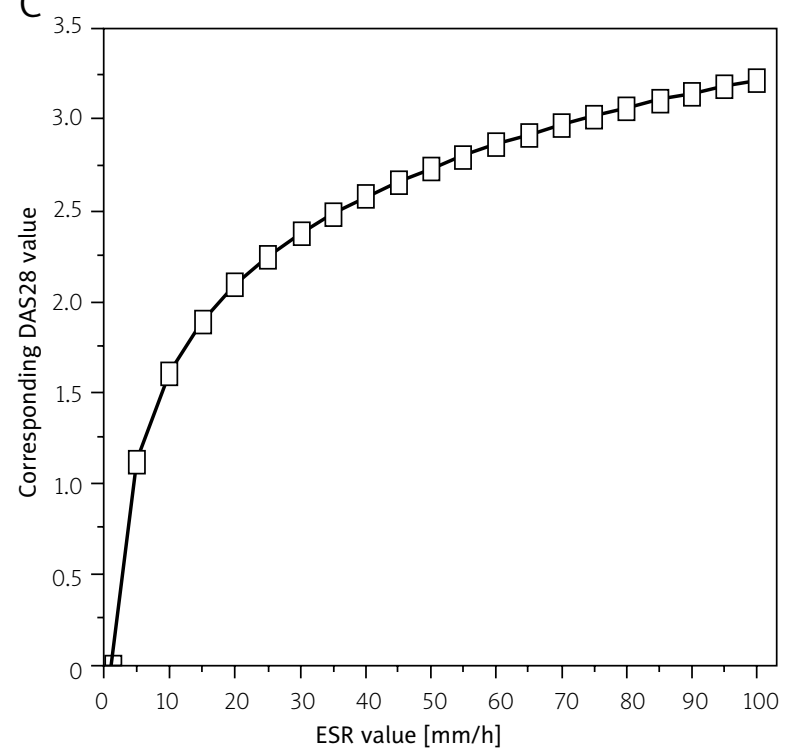

B

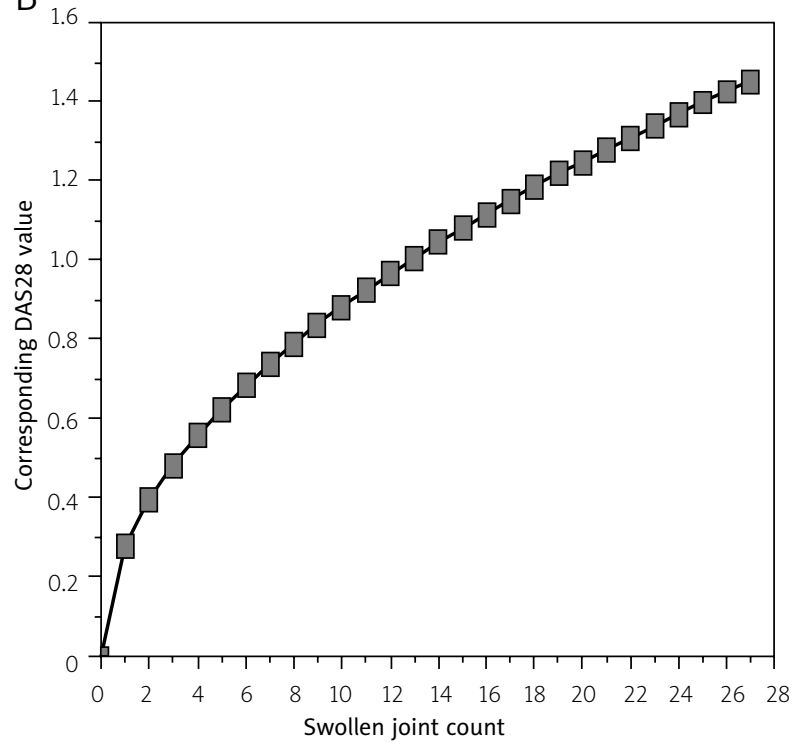

$\mathrm{D}$

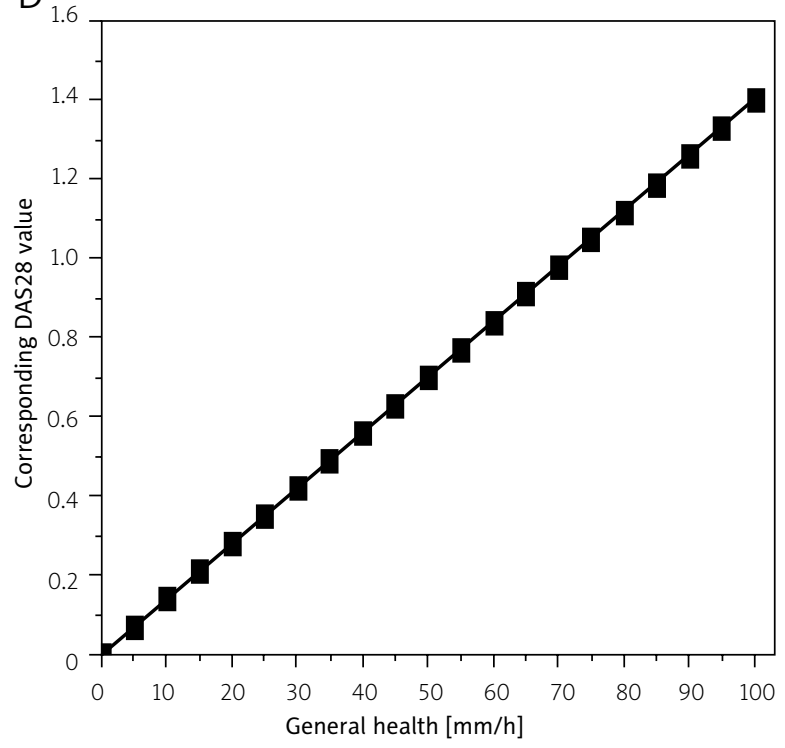

Fig. 1. Graphs of functions for the DAS28 score parameters: A) tender joint count function; B) swollen joint count function; C) ESR function; D) general health assessment function.

It is important to recognize that the use of ESR in the DAS28 score has limitations; as the ESR parameter is age and gender dependant (it increases with age and is higher in females) and is also dependant on blood cell count (it typically increases with anemia), this may lead to inaccurate reflection of disease activity [5].

The contribution of both tender and swollen joint count to the DAS28 score is expressed as a power function. Similar to the logarithmic function, this translates into a rapid increase in the score in the lower range (i.e. a smaller number of affected joints) and slowing of the increase in the score in the higher range. Therefore the effect of therapy on the score is much more pronounced when only a small number of joints are involved in the disease. From a clinical standpoint, this behavior of the function seems logical. For example, there would be a much more significant decrease in disease activity in a patient who had a drop in the tender/swollen joint count from 4 to 2 than in a patient with a drop from 10 to 8 joints.

A major drawback of the DAS28 score in the authors' opinion is related to the difference in the effects of tender and swollen joint counts. Based on formula [1] the effect of the number of tender joints is two-fold larger than the effect of the swollen joint count. This is explained by the fact that the parameter of swollen joint 
Table III. Effect of ESR level changes on DAS28

\begin{tabular}{|ccccc|}
\hline \multicolumn{2}{c}{ Start value } & \multicolumn{2}{c|}{ End value } & Difference in DAS28* \\
\cline { 1 - 3 } ESR $[\mathrm{mm} / \mathrm{h}]$ & DAS28 $^{*}$ & ESR $[\mathrm{mm} / \mathrm{h}]$ & DAS28 $^{*}$ & 0.13 \\
\hline 60 & 2.87 & 50 & 2.74 & 0.16 \\
\hline 50 & 2.74 & 40 & 2.58 & 0.20 \\
\hline 40 & 2.58 & 30 & 2.38 & 0.28 \\
\hline 30 & 2.38 & 20 & 2.10 & 0.49 \\
\hline 20 & 2.10 & 10 & 1.61 & \\
\hline
\end{tabular}

*only ESR value

count is dependent on the examiner's experience and thus it is less reproducible when compared to the tender joint count parameter.

It is important to understand that DAS/DAS28 scores were developed nearly 30 years ago, prior to the major advances in understanding the pathophysiology of RA which led to development of better therapeutic measures [6]. In addition, in the past 20 years there have been major advances in imaging techniques.

It is well known today that synovitis, followed by synovial edema and then hypertrophy, is the basis of RA [6]; and modern ultrasound machines are equipped with high resolution transducers, capable of assessing synovial inflammation.

Consequently, it would be useful to assess joints for swelling using ultrasound. This will likely lead to a more objective and reproducible swollen joint count and should be used for the DAS28 score in the authors' opinion.

An additional limitation of the DAS28 score is related to irreversible destructive joint lesions which often develop in the course of RA. Such joints remain tender even after the inflammatory process subsides. The DAS28 score is significantly affected by the parameter of tender joint count, which in this case will lead to overestimation of disease activity and in extreme cases may falsely determine therapy as ineffective in accordance with EULAR criteria.

In summary, in light of recent years' advances it appears that the DAS28 may not reflect disease activity

Table IV. Comparison of tender and swollen joint counts on DAS28

\begin{tabular}{|ccc|}
\hline $\begin{array}{c}\text { Number of } \\
\text { tender joints }\end{array}$ & $\begin{array}{c}\text { Number of } \\
\text { swollen joints }\end{array}$ & DAS28 $^{*}$ \\
\hline 2 & 8 & 0.79 \\
\hline 4 & 16 & 1.12 \\
\hline 6 & 24 & 1.37 \\
\hline
\end{tabular}

*only tender/swollen joint value accurately due to several limitations, of which in the authors' opinion the most significant is the high weight of the parameter of swollen joint count in the score, as this parameter may not be determined accurately based on physical examination. This parameter may be assessed more accurately with ultrasonography (US), which may lead to improvement in the accuracy of the DAS28 score in determining disease activity and response to therapy.

\section{DAS28 - therapy monitoring}

In therapy monitoring, the effect of individual parameters on the score change is strongly dependent on their initial values in the case of tender and swollen joint counts, and ESR. This relationship is clearly observed when evaluating the changes in the value of ESR.

Table III presents the change of ESR-dependent function of DAS28 score for some examples of ESR values seen in clinical practice and change in DAS28 with an ESR decrease of $10 \mathrm{~mm} / \mathrm{h}$.

The results in Table III clearly show that reductions in the ESR value in similar increments will result in modest changes in the DAS28 score when the baseline ESR is high and in a significant score change when the baseline ESR is low.

When considering tender and swollen joint counts, the effect of the baseline value on the change in the score is less significant than with ESR.

The data in Table IV demonstrate the higher significance of tender joint count change over swollen joint count change. As swollen joint count number change must be four times higher than the tender joint count change to produce the same effect on the DAS28 score. This may lead to inaccurate assessment of disease activity, as discussed previously.

\section{Rheumatoid arthritis activity assessment by ultrasonography}

Recent scientific reports suggest that imaging methods, particularly US, should play a more important role in RA therapy monitoring and activity evaluation. 
This led to the development of numerous new score systems which use US as a main tool for RA activity assessment. The most notable in the authors' opinion is the SAS 1 score developed by Grassi et al. This score is determined by evaluation of a single joint: the metacarpophalangeal (MCP) joint, with the most florid synovitis. This is based on the assumption that this would be sufficient for monitoring changes in RA activity during treatment [7]. Examining a single joint in order to evaluate changes in RA activity may raise concerns with regard to the accuracy of assessment. Nonetheless, one should take into account that MCP joints are among the most frequent early targets of RA [7]. While the SAS 1 score still needs to be compared to other US scoring systems, it demonstrates that US examination will likely play a major role in the disease assessment in the future.

Ultrasonography is currently often used in clinical studies to assess the effect of biologic drugs in monotherapy or in combination with methotrexate [8, 9]. Based on the current understanding of RA pathophysiology, it is widely accepted that synovitis containment is a significant contributor to therapy efficacy.
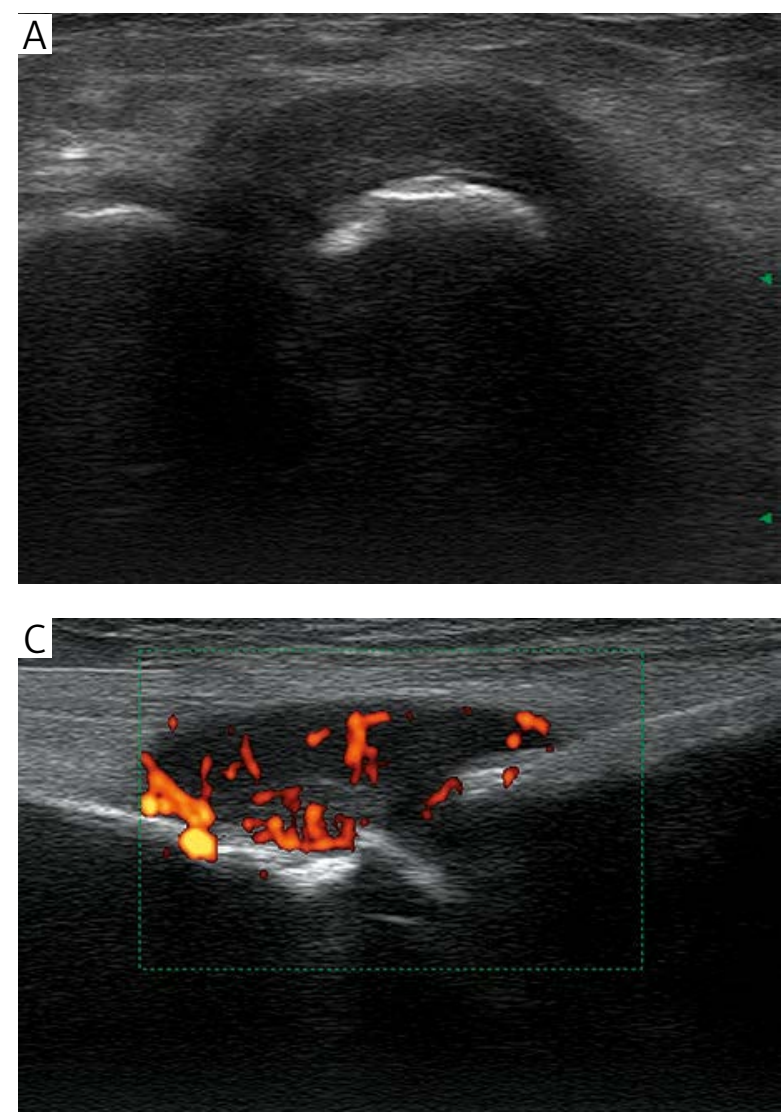

US enables follow-up of early RA changes. However, this examination remains highly dependent on the operator's experience. This is best demonstrated in Fig. 2, which shows the effect of transducer pressure on synovial perfusion. Any attempt to include US in diagnostic criteria would require further training of rheumatologists and sonographers in US scanning that would permit more common use of US as a criterion for RA therapy effectiveness monitoring.

Nevertheless, prior studies suggest that use of US by experienced sonographers, especially power Doppler ultrasonography (PD US), as a tool for synovitis evaluation leads to much better outcomes than when relying on radiographs [8-10].

\section{Summary}

In summary, based on the authors' opinion and experience, the use of the SAS 1 score yields much more objective results than DAS28, which is biased by subjectivity due to use of patient's self-assessed general health and by evaluation of tender and swollen joint counts.
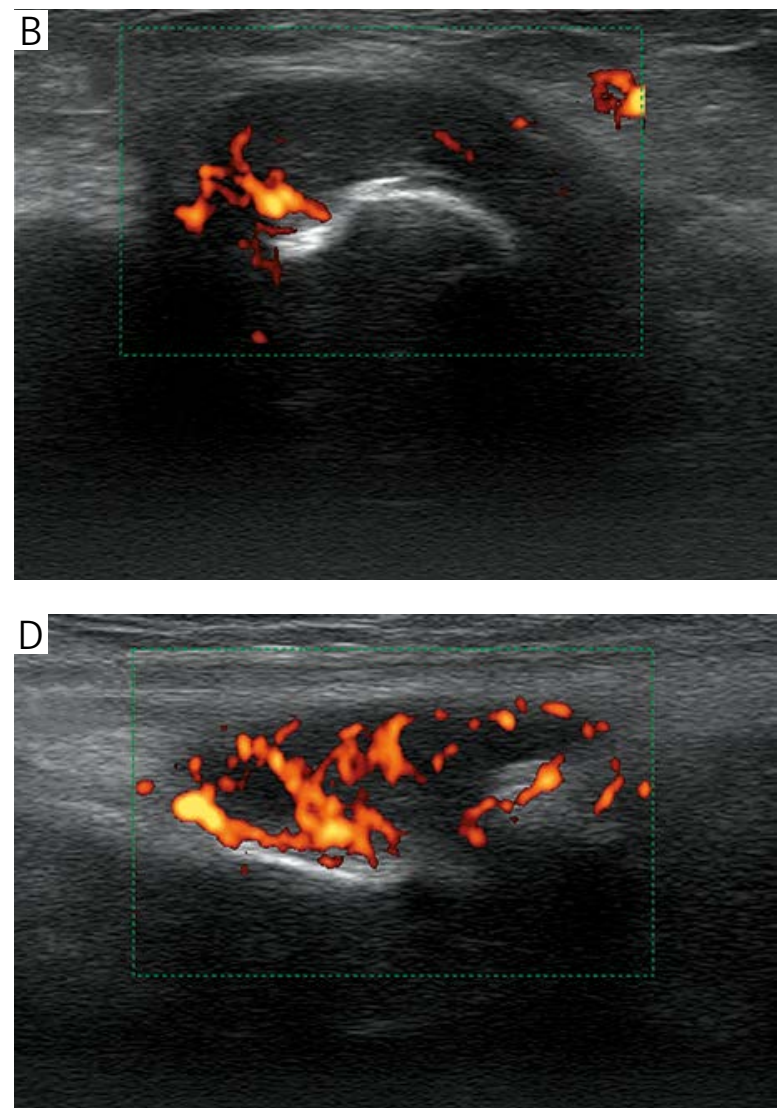

Fig. 2. Effect of transducer pressure on synovial perfusion in PD US in a patient with active RA; A) heavy pressure - no perfusion (grade 0); B) medium pressure - slight synovial hyperemia (grade 1); C) light pressure - moderate synovial hyperemia (grade 2); D) proper transducer handling - marked synovial hyperemia (grade 3). 
Using the SAS 1 score provides results of remission more often than the DAS28 score, as synovitis subsidence is more easily appreciated on US, while in the same patients the DAS28 score may remain increased due to elevated ESR caused by old age or due to irreversibly damaged joints which remain tender.

Nevertheless, despite many attempts to develop an imaging-based management algorithm, DAS/DAS28, due to the relative ease of its acquisition and calculation, still remains a good tool for assessment of disease activity, particularly at the early stage of RA, and is the most widely used accessory tool for clinical purposes.

The authors declare no conflict of interest.

\section{References}

1. van Riel PL. Provisional guidelines for measuring disease activity in clinical trials on rheumatoid arthritis. Br J Rheumatol 1992; 31: 793-796.

2. Frasen J, van Riel P. Disease Activity Score and the Eular response criteria. Clin Exp Rheumatol 2005; 23 (3 suppl. 39): 93-99.

3. Wislowska M, Kalinska I, Olczyk-Kwiecień B. Stare i nowe metody oceny aktywności choroby, stopnia uszkodzenia tkanek i utraty funkcji w reumatoidalnym zapalaniu stawów. Problemy Lekarskie 2006; 45: 52-56.

4. Magnani M, Salizzoni E, Mulè R, et al. Ultrasonography detection of early bone erosions in the metacarpophalangeal joints of patients with rheumatoid arthritis. Clin Exp Rheumatol 2004; 22: 743-748.

5. Jurado RL. Why shouldn't we determine the erythrocyte sedimentation rate? Clin Infect Dis 2001; 33: 548-549.

6. Brown AK, Quinn MA, Karim Z, et al. Presence of significant synovitis in rheumatoid arthritis patients with disease-modifying antirheumatic drug-induced clinical remission: evidence from an imaging study may explain structural progression. Arthritis Rheum 2006; 54: 3761-3773.

7. Grassi W, Gaywood I, Pande I, et al. From DAS 28 to SAS 1 . Clin Exp Rheumatol 2012; 30: 649-651.

8. Naredo E, Möller I, Cruz A, et al. Power Doppler ultrasonographic monitoring of response to anti-tumor necrosis factor therapy in patients with rheumatoid arthritis. Arthritis Rheum 2008; 58: 2248-2256.

9. Fiocco U, Ferro F, Vezzù M, et al. Rheumatoid and psoriatic knee synovitis: clinical, grey scale, and power Doppler ultrasound assessment of the response to etanercept. Ann Rheum Dis 2005; 64: 899-905.

10. Ikeda K, Nakagomi D, Sanayama Y, et al. Correlation of radiographic progression with the cumulative activity of synovitis estimated by power Doppler ultrasound in rheumatoid arthritis: difference between patients treated with methotrexate and those treated with biological agents. J Rheumatol 2013; 40: 1967-1976. 